\title{
Subungual Metastasis of an Adenocarcinoma of the Prostate in a Finger
}

\author{
Ramón García-Galaviz ${ }^{\mathrm{a}}$ Judith Domínguez-Cherit ${ }^{\mathrm{a}} \quad$ Claudia Caro-Sánchez $^{\mathrm{b}}$ \\ Elizabeth Salazar-Rojas ${ }^{\mathrm{C}}$ \\ aDermatology Department, Instituto Nacional de Ciencias Médicas y Nutrición Salvador Zubirán, Mexico City,

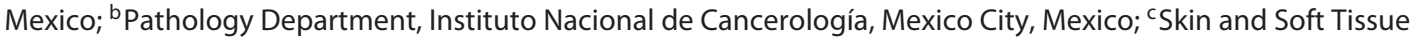 \\ Department, Instituto Nacional de Cancerología, Mexico City, Mexico
}

\section{Established Facts}

- Subungual metastasis is a very rare condition with a poor prognosis.

\section{Novel Insights}

- This is the first case report of an adenocarcinoma of the prostate with subungual metastasis in a finger.

\section{Keywords}

Adenocarcinoma of the prostate - Dermatologic surgery · Dermoscopy $\cdot$ Nail bed tumor · Nail tumor · Subungual metastasis

\begin{abstract}
Cutaneous metastasis is a rare event occurring most frequently in patients previously diagnosed with cancer. However, subungual metastases are even less frequent. The most common neoplasms associated with the latter are lung, kidney, and breast neoplasms. Lung cancer is the main cause of subungual metastases in the fingers, and genitourinary tract tumors are the main cause of subungual metastases in the toes. This is the first case report of an adenocarcinoma of the prostate with subungual metastasis.

(c) 2018 S. Karger AG, Basel
\end{abstract}

\section{Introduction}

Cutaneous metastases are defined as neoplastic lesions affecting the skin that originate from a primary tumor at a different site. They are rarely the initial presentation of a previously undiagnosed tumor and typically occur in patients with an established diagnosis of malignancy and, therefore, indicate either progression or recurrence of the cancer [1].

\section{Case Report}

We present the case of a 66-year-old male diagnosed with metastatic cribriform adenocarcinoma of the prostate 2 years earlier. He was treated with bicalutamide and tamsulosin. During followup, prostate-specific antigen levels rose from 95 to $191 \mathrm{ng} / \mathrm{dL}$ and

\section{KARGER}

(c) 2018 S. Karger AG, Basel

E-Mail karger@karger.com

www.karger.com/sad
Dr. Elizabeth Salazar-Rojas

Instituto Nacional de Cancerología

Av. San Fernando 22, Belisario Domínguez Sección XVI

Tlalpan, Mexico City 14080 (Mexico)

E-Mail dra.elisalazar@gmail.com 
Fig. 1. a A painful enlargement on the nail plate of the fourth right finger, with violaceous discoloration, without destroying the nail plate. $\mathbf{b}$ Onychoscopy showed nonmelanocytic black pigmentation of the nail bed, with hematomas and vascular-like nodules, as well as dilated blood vessels. The nail plate was unremarkable.
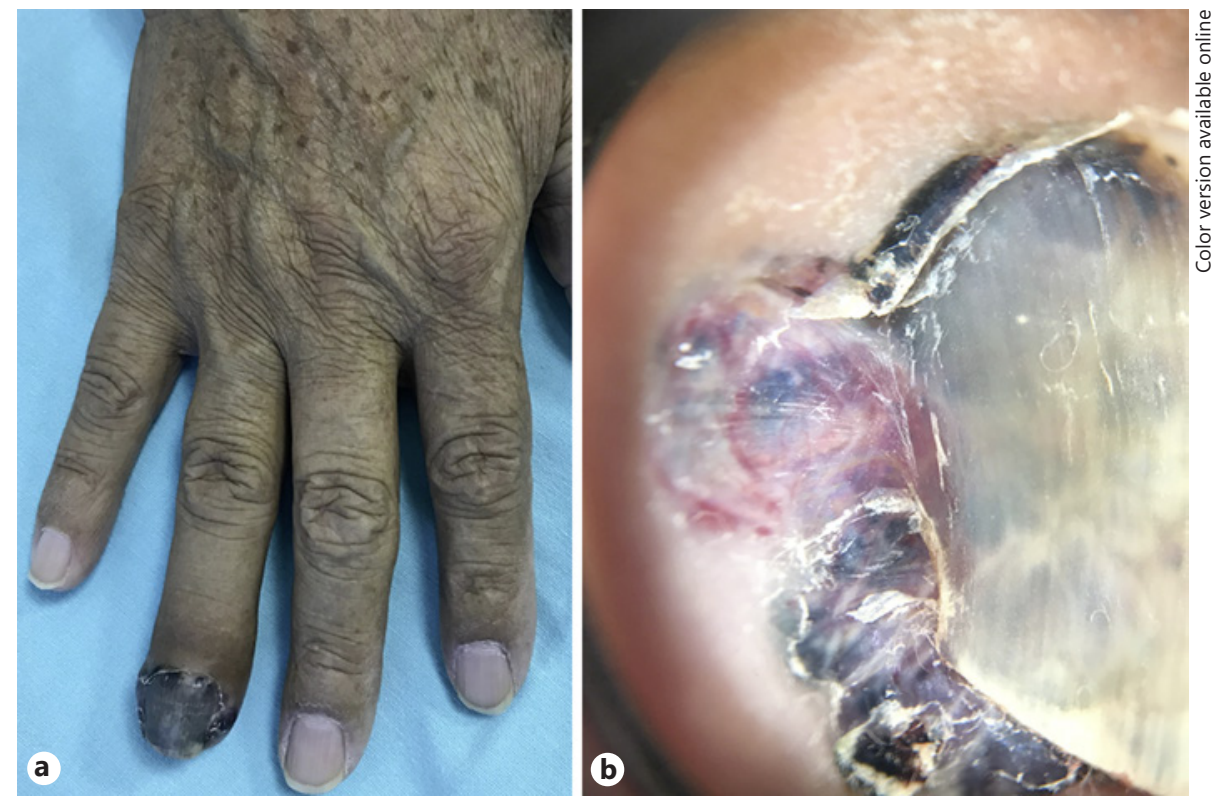

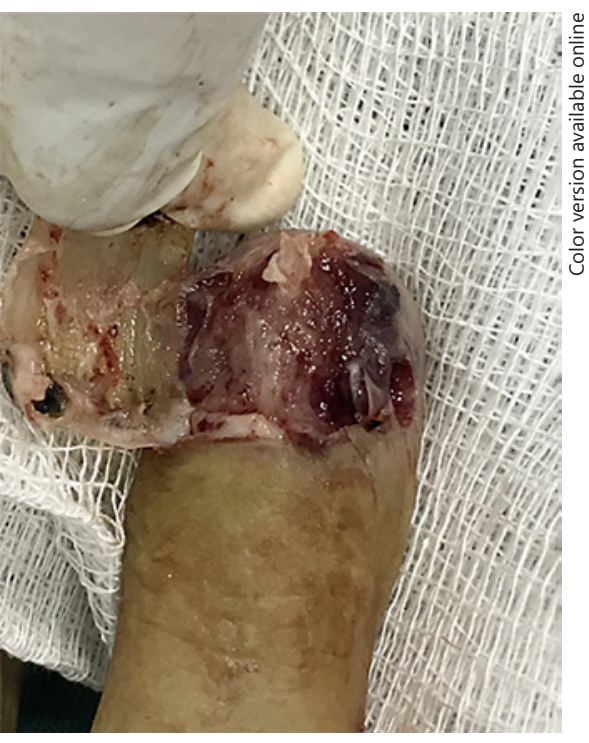

Fig. 2. Partial avulsion of the nail plate was performed showing a friable, poorly defined tumor with many vascular-like and purpuric areas.

free prostate-specific antigen from 17 to $>30 \mathrm{ng} / \mathrm{dL}$. A painful enlargement on the nail plate of the fourth right finger was also noted, with violaceous discoloration, without destroying the nail plate (Fig. 1a). Onychoscopy showed nonmelanocytic black pigmentation of the nail bed, with hematomas and vascular-like nodules, as well as dilated blood vessels. The nail plate was unremarkable (Fig. 1b).

Subungual Metastasis of an

Adenocarcinoma of the Prostate
A digital nerve block and a tourniquet were applied in the fourth finger. Partial avulsion of the nail plate was performed showing a friable, poorly defined tumor with many vascular-like and purpuric areas (Fig. 2). A 4-mm punch was taken from the nail bed. Histopathology showed the neoplasia affecting the nail bed, with a cribriform pattern (Fig. 3a) and rigid lumina of varying size (Fig. 3b). High-power view demonstrated cuboid cells with an ovoid nucleus, granular chromatin, and prominent nucleolus (Fig. 3c). Mitoses were present. Immunostain was diffusely positive for prostate-specific antigen (Fig. 3d).

On follow-up, metastatic disease was found on many sites such as bone, liver, and rectum. The disease was castrate resistant, and docetaxel-based chemotherapy was initiated, but disease progression was noted, and the patient died 6 months after evaluation.

\section{Discussion}

Cutaneous metastases may precede, accompany, or follow the diagnosis of the primary tumor. They are more often found in older patients and classically present as multiple painless nodules near the primary site. It is a rare event occurring only in $5-10 \%$ of patients already diagnosed with any kind of cancer, and $0.8-3.5 \%$ occur as the first sign of malignancy [2]. Most skin metastases manifest at end-stage disease of the primary neoplasm or during treatment [3]. They are more common in men, and the mean age of presentation is 55 years [4].

Although the biomolecular basis of metastasis is complex and poorly understood, there are 3 basic patterns of metastatic mechanisms reported: (1) mechanical tumor 

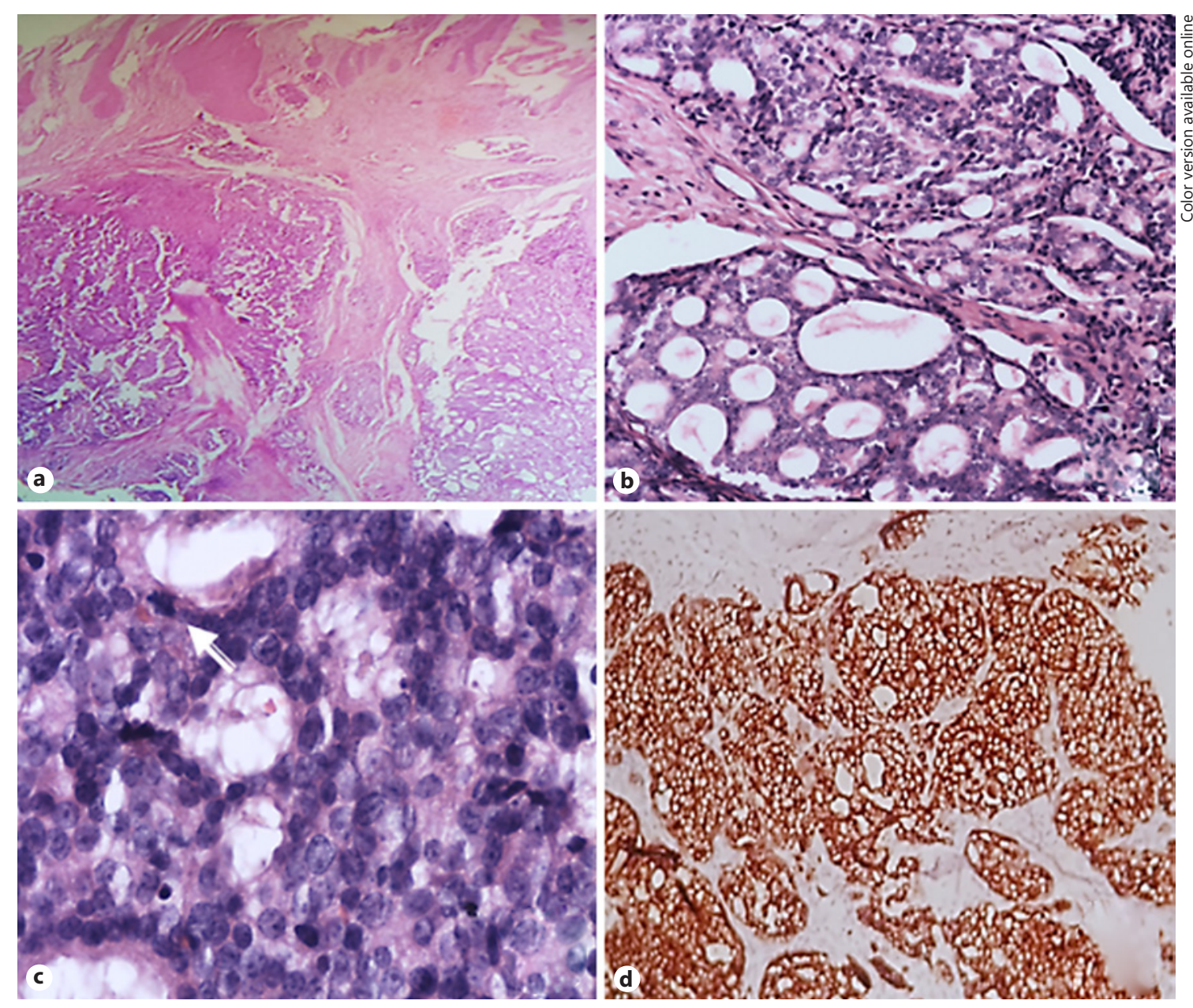

Fig. 3. Microphotographs show the neoplasia affecting the nail bed, with a cribriform pattern $(\mathrm{HE}, \times 100)(\mathbf{a})$ and rigid lumina of varying size $(\mathrm{HE}, \times 200)(\mathbf{b})$. c High-power view demonstrated cuboid cells with an ovoid nucleus, granular chromatin, and prominent nucleolus $(\mathrm{HE}, \times 400)$; mitoses were present (arrow). d Immunostain was diffusely positive for prostate-specific antigen.

stasis: anatomical proximity and lymphatic drainage; (2) organ-specific factors: selective affinity of tumor cells to a specific organ; and (3) nonselective factors: independent of mechanical and organ-specific factors [5].

Metastases affecting the nail apparatus, also called acral or subungual metastases, are extremely rare. They represent only $0.1 \%$ of cutaneous metastases. The most common cause is direct extension from a bony lesion beneath the nail plate. In contrast to cutaneous metastasis, subungual metastasis may be the initial manifestation of a previously undiagnosed cancer in $44 \%$ of patients [6].

The clinical findings should be distinguished from other nail diseases, such as infections, inflammatory dermatoses, primary nail tumors (benign and malignant), and, as in our case, traumatic hematomas. It may be possible to identify an erythematous-violaceous nodule below the nail plate (the most frequent location), and periungual lesions may be the result of direct extension of subungual metastasis. The symptoms will depend on the tumor extension, infiltration to the nail bed, and bone invasion, ranging from an asymptomatic to a very painful nail, most commonly the latter. The most frequent location is a single finger, but either a single digit of the foot or multiple digits of the same hand or foot and/or both hands or feet have been described previously.

In a review of the literature, Cohen [6] reported 133 patients with subungual metastases, with the lung as the main primary tumor site (41\%), followed by the kidney
48

Skin Appendage Disord 2019;5:46-49 DOI: $10.1159 / 000489279$
García-Galaviz et al. 
(11\%) and breast (9\%). No primary tumors of the prostate were reported in this review. Lung cancer was the main cause of subungual metastases in the fingers, and genitourinary tract tumors were the main cause of subungual metastases in the toes.

The diagnosis should be confirmed through histopathologic and radiologic examination. X-ray films will show osteolysis of the affected phalange, while the joint cartilage and space are spared [7]. Histopathologic examination will reveal tumor cells from another primary neoplasm and, sometimes, will require immunohistochemistry in order to clarify the origin of tumor cells.

Treatment of subungual metastases should be tumor directed, either local therapies or incorporated into systemic treatment, or both. Systemic chemotherapy, nail avulsion and curettage of the tumor, excision of the tumor, amputation of the digit containing the tumor, and radiotherapy have been used.
The presence of cutaneous metastasis is a sign of advanced disease and therapeutic fail and/or recurrence, and survival will depend on the primary tumor type. If there is acral or nail apparatus involvement, the longest survival reported was 14 months.

To our knowledge, this is the first case report of an adenocarcinoma of the prostate with subungual metastasis as a sign of disease progression, with poor prognosis.

\section{Statement of Ethics}

The patient gave his informed consent for the use of anonymized photographs for research purposes.

\section{Disclosure Statement}

The authors declare no conflicts of interest.

\section{References}

Subungual Metastasis of an Adenocarcinoma of the Prostate
1 García-Arpa M, Rodríguez-Vázquez M, Sánchez-Caminero P, Delgado M, Vera E, Romero G, et al: Metástasis digital acral. Actas Dermosifiliogr 2006;97:334-336.

2 Chang P, Rosales D, Calderón G, GarcíaAceituno LF: Metástasis de un adenocarcinoma ductal invasivo al aparato ungueal. Dermatología CMQ 2013;11:257-260.

3 Cho EY, Kim TH, Park SD, Yun KJ, Choi SC, Kim HC, Nah YH: Acral metastasis in a patient with ampullary carcinoma. Korean J Intern Med 2007;22:55-58.

4 Vine JE, Cohen PR: Renal cell carcinoma metastatic to the thumb: a case report and review of subungual metastases from all primary sites. Clin Exp Dermatol 1996;21:377-380.

5 Weimann ET, Botero EB, Mendes C, Santos MA, Stelini RF, Zelenika CR: Cutaneous metastasis as the first manifestation of occult malignant breast neoplasia. An Bras Dermatol 2016;91(5 suppl 1):105-107.

6 Cohen PR: Metastatic tumors to the nail unit: subungual metastases. Dermatol Surg 2001; 27:280-293.

7 Gallagher B, Yousef G, Bishop L: Subungual metastasis from a rectal primary: case report and review of the literature. Dermatol Surg 2006:32:592-595. 